\title{
A dieta alimentar da presa Tenebrio molitor (Coleoptera: Tenebrionidae) pode afetar o desenvolvimento do predador Podisus nigrispinus (Heteroptera: Pentatomidae)?
}

\author{
Can the diet of the prey Tenebrio molitor (Coleoptera: Tenebrionidae) affect the \\ development of the predator Podisus nigrispinus (Heteroptera: Pentatomidae)?
}

\author{
Claubert Wagner Guimarães de Menezes ${ }^{1 *}$, Silma da Silva Camilo², Arley José Fonseca², \\ Sebastião Lourenço de Assis Júnior ${ }^{3}$, Diego Faustolo Bispo², Marcus Alvarenga Soares ${ }^{2}$
}

RESUMO: Inimigos naturais são importantes para o controle de pragas em culturas agrícolas e florestais. A criação de insetos predadores em biofábricas deve ser de baixo custo para serem utilizados em programas de Manejo Integrado de Pragas (MIP). O objetivo deste trabalho foi avaliar o desenvolvimento de Podisus nigrispinus Dallas, 1851 (Heteroptera: Pentatomidae), alimentado com larvas de Tenebrio molitor Linnaeus, 1758 (Coleoptera: Tenebrionidae), criadas com as seguintes dietas: farelo de trigo, ração triturada ou peletizada para aves poedeiras e fubá de milho. Foram obtidos os parâmetros de desenvolvimento e reproduçáo necessários para calcular a tabela de vida do predador. Os parâmetros da tabela de vida revelaram crescimento populacional em todos os tratamentos. No entanto, a taxa líquida de reprodução $(R o)$ de $P$. nigrispinus foi menor quando alimentados com larvas de $T$. molitor criadas com fubá de milho, mostrando ser a alimentação menos adequada para esse predador. Por proporcionar maior número total de ovos, o farelo de trigo constituiu a melhor dieta para P. nigrispinus. Estudos sobre dietas de presas alternativas são importantes, pois podem favorecer a nutrição de inimigos naturais e, consequentemente, melhorar o desempenho das criaçôes massais em laboratório.

PALAVRAS-CHAVE: Biologia; criação massal; controle biológico; inimigos naturais; nutrição de insetos.

\begin{abstract}
Natural enemies are important for controlling pests in agricultural and forestry culture. The reproductions of predatory insects in biofactories should have low cost to be used in Integrated Pest Management Program (IPM). The objective of this study was to evaluate the development of Podisus nigrispinus Dallas, 1851 (Heteroptera: Pentatomidae) fed with Tenebrio molitor Linnaeus, 1758 (Coleoptera: Tenebrionidae). The larvae of T. molitor were created with the following diets: wheat bran, shredded or pelleted poultry feed layers and corn meal. Parameters for the development and reproduction of the P. nigrispinus were obtained. Parameters of the life table show population growth in all treatments. However, the net rate of reproduction $(R o)$ of $P$. nigrispinus was lower when they were fed with larvae of $T$. molitor created with corn meal, which proved to be the least adequate food for this predator. By providing a larger total number of eggs, wheat bran was the best diet for $P$. nigrispinus. Studies on alternative prey diets are important as they may favor the nutrition of natural enemies and, consequently, improve the performance in laboratory rearing.
\end{abstract}

KEYWORDS: Biology; biological control; insect nutrition; mass rearing; natural enemies.

\footnotetext{
'Departamento de Fitotecnia; Universidade Federal de Lavras (UFLA) - Lavras (MG), Brasil.

${ }^{2}$ Departamento de Agronomia; Universidade Federal dos Vales do Jequitinhonha e Mucuri (UFVJM) - Diamantina (MG), Brasil.

${ }^{3}$ Departamento de Engenharia; UFVJM - Diamantina (MG), Brasil.

*Autor correspondente: claubertmenezes@yahoo.com.br

Recebido em: 19/11/2012. Aceito em: 16/02/2014
} 


\section{INTRODUÇÃO}

Insetos predadores são úteis para o controle natural de herbívoros, evitando que estes se tornem pragas (INAYAT et al., 2011; Neves et al., 2010; Menezes et al., 2012a). Podisus nigrispinus Dallas, 1851 (Heteroptera: Pentatomidae) é um percevejo encontrado em culturas florestais e agrícolas no Brasil, com eficiência em predar lepidópteros e coleópteros daninhos (TORRes et al., 2006; Moura; Grazia, 2011; Oliveira et al., 2011; Menezes et al., 2012b). Este predador tem sido criado em laboratório e liberado em programas de controle biológico em culturas como o eucalipto (ZANUNCio et al., 2002a), algodáo (Medeiros et al., 2000), soja (Ferreira et al., 2008) e tomate (Torres et al., 2002).

O custo de criaçáo de inimigos naturais é um desafio para a comercialização e uso desses insetos em programas de manejo integrado de pragas (De Bortoli et al., 2011). É necessário estabelecer métodos de criação massal que tenham baixo custo, fácil manutenção e que forneçam nutrição adequada às espécies utilizadas no controle biológico. O tipo de dieta, seja ela artificial ou natural, pode influenciar no desenvolvimento do predador e afetar seu desempenho reprodutivo (Lemos et al., 2003). O percevejo predador $P$. nigrispinus geralmente é alimentado com larvas ou pupas de Tenebrio molitor Linnaeus, 1758 (Coleoptera: Tenebrionidae) devido ao baixo custo e fácil manutenção em laboratório (Vivan et al ., 2002). Estas podem afetar o desempenho reprodutivo deste predador, podendo ser de forma semelhante (Lemos et al., 2003) ou superior a presas naturais, como larvas de Lepidoptera (Moreira et al., 1998; Espindula et al., 2010) ou presas alternativas, como o bicho da seda (ZANuncio et al., 1994) ou larvas de mosca doméstica (Jusselino Filho et al., 2001). Esta última, no entanto, pode ter custo mais baixo (De Bortoli et al., 2011).

Além da criação de $P$. nigrispinus, larvas, pupas e adultos de T. molitor podem ser utilizados na criação de outros predadores (Grundy et al., 2000; ZANuncio et al., 2004), parasitoides (ZANuncio et al., 2008; ANDRADE et al., 2010), pássaros (Martinson; Flaspohler, 2003), morcegos (MCCartney et al., 2007), aranhas (JaPYAssú; JotTA, 2005), peixes (NG et al., 2001) e lagartos (FonT; Molina-BorJa, 2004) e na degradação de resíduos de criatórios de aves (Fischer et al., 2004).
Estudos sobre o potencial dos predadores no controle de pragas-chave devem priorizar sua criação massal em laboratório, para que possam ser posteriormente liberados no campo e integrados ao sistema de manejo de pragas (Oliveira et al., 2004).

Tenebrio molitor é uma praga típica de farináceos. A dieta de criação de T. molitor pode influenciar o seu desenvolvimento (Morales-Ramos et al., 2010; 2011) e, possivelmente, o desempenho dos predadores que se alimentam desta espécie. Embora a dieta à base de farelo de trigo seja a mais utilizada para a sua criação (ZAMPERLINI et al., 1992), alguns criadores de pássaros têm usado dietas alternativas, como raçóes para aves poedeiras.

O objetivo deste trabalho foi avaliar, por meio de tabela de vida, o desenvolvimento de P. nigrispinus alimentado com larvas de T. molitor criadas em diferentes dietas.

\section{MATERIAL E MÉTODOS}

O experimento foi conduzido em câmara climatizada tipo BOD, a $25 \pm 2^{\circ} \mathrm{C}$, umidade relativa de $70 \pm 10 \%$ e fotoperíodo de 12 horas (2000 lúmens), no Laboratório de Entomologia da Universidade Federal dos Vales do Jequitinhonha e do Mucuri (UFVJM), Diamantina, Minas Gerais, Brasil.

Tenebrio molitor foi obtido da criação mantida no laboratório de Entomologia da UFVJM, alimentado com farelo de trigo (Zamperlini, et al., 1992) e colmos de cana de açúcar (Saccharum spp. L.) ou fatias de chuchu (Sechium edule) para complementar a alimentaçáo e suprir líquidos (Fraenkel et al., 1950).

Larvas recém-eclodidas foram transferidas para quatro bandejas plásticas com diferentes dietas, constituindo os seguintes tratamentos: T1: farelo de trigo integral (controle); T2: raçáo peletizada para aves poedeiras da marca Extraovo $16 \mathrm{Guabi}^{\circledR}$; T3: ração farelada para aves poedeiras da marca Posturave $\mathrm{Guabi}^{\circledR}$; eT4: fubá de milho da marca Sinhá (Tabela 1). As larvas foram mantidas nesses recipientes até o último estágio, para serem utilizadas como presa para $P$. nigrispinus. A cada tratamento foi adicionada uma fatia de chuchu, trocada semanalmente, para fornecer umidade às larvas.

Tabela 1. Composição nutricional das dietas fornecidas a Tenebrio molitor.

\begin{tabular}{lcccc} 
Componentes & Farelo de trigo ${ }^{1}$ & $\begin{array}{c}\text { Ração para aves de } \\
\text { postura (peletizada) }\end{array}$ & $\begin{array}{c}\mathbf{R}^{1} \\
\text { Ração para aves de } \\
\text { postura (farelada) }\end{array}$ & Fubá de milho' $^{{ }^{1}}$ \\
\hline Umidade & $13,50 \%$ & $13,00 \%$ & $13,00 \%$ & $12,52 \%$ \\
\hline Proteína bruta & $13,00 \%$ & $16,00 \%$ & $17,00 \%$ & $7,88 \%$ \\
\hline Lipídeos & $2,00 \%$ & $2,50 \%$ & $2,00 \%$ & $3,65 \%$ \\
\hline Fibra bruta & $11,00 \%$ & $6,00 \%$ & $6,00 \%$ & $1,73 \%$ \\
\hline Matéria mineral & $6,50 \%$ & $16,00 \%$ & $15,00 \%$ & $1,27 \%$ \\
\hline Fósforo & $1,04 \%$ & $0,50 \%$ & $0,55 \%$ & $0,06 \%$ \\
\hline Cálcio & $0,10 \%$ & $4,20 \%$ & $4,20 \%$ & $0,03 \%$ \\
\hline Aflatoxinas & $20,00 \mathrm{ppb}$ & - & - & - \\
\hline
\end{tabular}

'Valores encontrados nos rótulos dos produtos. 
As ninfas de P. nigrispinus utilizadas nos testes foram obtidas da criação do Laboratório de Entomologia da UFVJM. O delineamento utilizado foi o inteiramente casualizado (DIC), e os tratamentos foram constituídos por 10 repetiçóes, com 10 ninfas cada, em potes plásticos de $500 \mathrm{~mL}$. A partir do segundo estádio as ninfas foram alimentadas, diariamente, com duas larvas de T. molitor tratadas com as diferentes dietas. $\mathrm{O}$ fornecimento de água destilada às ninfas foi feito por um tubo de vidro, tipo anestésico.

Foram confeccionadas tabelas de vida para avaliação dos parâmetros de crescimento da população de $P$. nigrispinus (Southwood, 1978). Os parâmetros avaliados foram a taxa líquida de reprodução $(R o)$ (número de fêmeas adicionadas por fêmea durante sua vida), $R o=\sum(\mathrm{mx} . \mathrm{Lx})$; tempo médio de geração $(T)$ (tempo entre o nascimento dos pais e dos filhos) $T=\left(\sum \mathrm{mx} . \mathrm{lx} . \mathrm{x}\right) /\left(\sum \mathrm{mx} . \mathrm{lx}\right)$; razão infinitesimal de aumento $(R m)$ (taxa de aumento populacional por unidade de tempo) $R m=\ln \mathrm{Ro} / \mathrm{T}$; razão finita de aumento $(\lambda)$ (número de fêmeas adicionadas à população por fêmea desse predador por unidade de tempo) $\lambda=\mathrm{e}^{\mathrm{rm}}$; e $(T D)$ (tempo necessário para o predador dobrar sua população em número de indivíduos) $T D=\ln (2) / \mathrm{rm}$ (MaIA et al., 2000).

Foram avaliados os dados reprodutivos de P. nigrispinus: número de ovos por postura e por fêmea, viabilidade e período embrionário, período de pré-oviposição, oviposição e pós-oviposição, e longevidade dos adultos machos e fêmeas.
As médias dos parâmetros da tabela de vida e reprodutivos foram submetidas aos testes de Bartlett e Kolmogorov para constatação de normalidade e homogeneidade da variância e, em seguida, à análise de variância e comparação pelo teste de Tukey a 5\%. Os dados foram analisados com o programa SAEG, Sistema para Análises Estatísticas, Versão 9.1, Universidade Federal de ViçosaUFV (SAEG, 2007).

\section{RESULTADOS}

A taxa líquida de reprodução $(R o)$ e o tempo médio de geração (T) de P. nigrispnus foram menores com o fubá de milho, mas semelhantes para os demais tratamentos (Tabela 2).

A razão infinitesimal $(R m)$, a razão finita de aumento populacional $(\lambda)$ e o tempo de duplicação da população em número de indivíduos $(T D)$ foram semelhantes e indicaram crescimento da população de $P$. nigrispinus em todos os tratamentos (Tabela 2).

A dieta à base de farelo de trigo proporcionou maior número total de ovos, além de maior número de posturas e período de oviposição mais longo, porém, semelhante ao da ração peletizada. Os demais parâmetros não variaram significativamente entre os tratamentos (Tabela 3).

Tabela 2. Parâmetros da tabela de vida de Podisus nigrispinus (Heteroptera: Pentatomidae) alimentado com larvas de Tenebrio molitor (Coleoptera: Tenebrionidae) submetido a diferentes dietas de criação $\left(25 \pm 2{ }^{\circ} \mathrm{C}, 70 \pm 10 \%\right.$ UR e fotoperíodo de 12 horas).

\begin{tabular}{lccccc} 
Trat $^{1}$ & Ro & $T$ & $R m$ & $\lambda$ & $T$ \\
T1 & $254,6 \pm 112,0 a$ & $62,2 \pm 7,9 a$ & $0,09 \pm 0,01 a$ & $1,09 \pm 0,02 a$ & $7,9 \pm 1,26 a$ \\
\hline T2 & $192,5 \pm 57,2 a$ & $59,7 \pm 6,7 a$ & $0,09 \pm 0,01 a$ & $1,09 \pm 0,01 a$ & $7,9 \pm 0,77 a$ \\
\hline T3 & $178,1 \pm 52,6 a$ & $54,4 \pm 4,8 a$ & $0,1 \pm 0,01 a$ & $1,10 \pm 0,01 a$ & $7,3 \pm 0,71 a$ \\
\hline T4 & $155,3 \pm 27,6 b$ & $53,1 \pm 5,8 b$ & $0,1 \pm 0,01 a$ & $1,10 \pm 0,01 a$ & $7,3 \pm 0,74 a$ \\
\hline
\end{tabular}

Médias seguidas pela mesma letra por linha não diferem pelo teste de Tukey a $p=0,05$

Ro: taxa líquida de reprodução, T: tempo médio de geração, Rm: Razão infinitesimal, $\lambda$ : razão infinita de aumento populacional; TD: tempo de duplicação da população em número de indivíduos; T1: farelo de trigo integral; T2: ração peletizada para aves; T3: ração farelada para aves e T4: fubá de milho.

Tabela 3. Variáveis de reprodução de Podisus nigrispinus (Heteroptera: Pentatomidae) alimentado com larvas de Tenebrio molitor (Coleoptera: Tenebrionidade) criadas com diferentes dietas $\left(25 \pm 2{ }^{\circ} \mathrm{C}, 70 \pm 10 \%\right.$ UR e fotoperíodo de 12 horas).

\begin{tabular}{|c|c|c|c|c|c|}
\hline Variáveis & Farelo de trigo & Ração peletizada & Ração farelada & Fubá de milho & CV (\%) \\
\hline Pré-oviposição & $3,87 \pm 0,44 a$ & $5,50 \pm 1,38 a$ & $4,00 \pm 0,50 a$ & $5,12 \pm 0,94 a$ & 27,03 \\
\hline Oviposição & $63,00 \pm 6,00 a$ & $45,62 \pm 10,28 a b$ & $40,87 \pm 6,94 b$ & $44,25 \pm 8,00 a b$ & 30,43 \\
\hline Pós-oviposição & $3,37 \pm 2,47 a$ & $3,50 \pm 2,25 a$ & $3,62 \pm 2,13 a$ & $3,75 \pm 2,06 a$ & 7,52 \\
\hline Número de Posturas & $29,50 \pm 4,88 a$ & $23,62 \pm 3,22 a b$ & $20,37 \pm 4,97 b$ & $18,87 \pm 4,63 b$ & 24,56 \\
\hline $\mathrm{N}^{\circ}$ Ovos por Postura & $27,36 \pm 2,56 a$ & $24,13 \pm 2,40 a$ & $32,53 \pm 2,28 a$ & $23,40 \pm 2,01 a$ & 34,52 \\
\hline $\mathrm{N}^{\circ}$ Total de Ovos & $805,75 \pm 154,25 a$ & $570,12 \pm 107,13 b$ & $508,87 \pm 109,01 b$ & $439,62 \pm 99,03 b b$ & 25,23 \\
\hline Período embrionário & $5,84 \pm 0,10 a b$ & $5,87 \pm 0,08 a$ & $5,90 \pm 0,13 a$ & $5,65 \pm 0,12 b$ & 2,47 \\
\hline Viabilidade dos Ovos & $83,97 \pm 4,90 a$ & $87,84 \pm 3,67 a$ & $88,18 \pm 2,06 a$ & $87,21 \pm 3,27 a$ & 6,65 \\
\hline Longevidade das Fêmeas & $76,75 \pm 16,00 a$ & $57,12 \pm 14,69 a b$ & $49,00 \pm 7,50 b$ & $59,25 \pm 14,75 a b$ & 29,75 \\
\hline Longevidade dos Machos & $57,87 \pm 17,41 a b$ & $36,62 \pm 12,28 b$ & $66,62 \pm 17,38 a$ & $64,25 \pm 6,25 a$ & 32,48 \\
\hline
\end{tabular}

Médias das variáveis seguidas pela mesma letra na linha não diferem significativamente pelo teste de Tukey a $5 \%$. 


\section{DISCUSSÃO}

Tabelas de vida sáo importantes para estudos do ciclo de vida de inimigos naturais, pois permitem avaliar o desenvolvimento e características reprodutivas em diferentes tratamentos, constituindo um meio simples e eficiente de reduzir o volume de dados e apresentá-los de forma facilmente analisável (Southwood, 1978). Elas podem determinar o aumento populacional das espécies ao mostrar o potencial reprodutivo de fêmeas nos diferentes tempos (Medeiros et al., 2000).

A qualidade da dieta oferecida às formas imaturas tem grande influência no desempenho reprodutivo de adultos de diversos predadores (FInKe, 2002; MaYnTZ et al., 2003; Michaud, 2000).

O teor de nutrientes na dieta de T. molitor como, caseína, glicose, colesterol, levedura, hidratos de carbono e proteínas são importantes para seu desenvolvimento (FraENKEL et al., 1950; URREjola et al., 2011). A menor taxa líquida de reproduçáo $(R o)$ de $P$. nigrispinus alimentado com larvas desenvolvidas em fubá de milho pode ser causada pelos baixos teores minerais e fibras e, principalmente, proteínas disponíveis nesta dieta (Tabela 1). Isso pode ter afetado o balanço nutricional de T. molitor, com reflexos negativos na alimentação do predador e, consequentemente, no seu desenvolvimento. Além disso, a alimentaçáo das larvas pode influenciar a ocorrência de determinadas enzimas, como a trealase, que promove a quebra do dissacarídeo trealose liberando glicose, importante nos gastos energéticos dessa presa (Lopes; Villela, 1972).

De Bortoli et al. (2011) encontraram que a presa mais adequada para a criação de $P$. nigrispinus é a lagarta da cana Diatreae saccharalis Fabricius, 1794 (Lepidoptera: Crambidae), com o maior valor de Ro entre as presas testadas, inclusive T. molitor. No entanto, esses valores foram inferiores ao encontrado neste trabalho, indicando a eficiência desta presa na criação de $P$. nigrispinus. Este é um dos principais parâmetros de uma tabela de vida, pois permite avaliar o crescimento populacional, envolvendo os valores da fertilidade específica das fêmeas nas diferentes idades (Southwood, 1978). Os valores de Ro revelaram crescimento populacional em todos os tratamentos. No entanto, o menor valor deste parâmetro para fubá de milho indica a não adequação desta dieta na criaçáo da presa, visando à produçáo massal de percevejos predadores.

A dieta composta por fubá de milho, embora fosse a menos adequada, promoveu o menor valor para o tempo de geração $(T)$. Este parâmetro indica o tempo decorrido desde o nascimento dos pais até o de seus descendentes, ou seja, o intervalo compreendido entre a postura de um ovo por uma fêmea de uma população até a de outro por uma fêmea procedente do primeiro, ou, ainda, o tempo médio em que uma geração é produzida (Southwood, 1978). Valores baixos deste parâmetro são desejáveis em programas de controle biológico, pois quanto menor esse valor, maior a possibilidade de se obter mais geraçóes anuais, o que proporciona vantagens financeiras. Já o tempo para duplicar a população $(T D)$ foi semelhante entre os tratamentos (Tabela 2). No entanto, os parâmetros que promovem prole mais numerosa $(R o, r m e \lambda)$ são mais relevantes que os de duração em termos de aumento populacional.

Os parâmetros razão infinitesimal $(\mathrm{rm})$ e a taxa finita de aumento populacional $(\lambda)$ foram semelhantes entre os tratamentos. Estes resultados foram diferentes dos encontrados por De Bortoli et al. (2011), em que o tipo de presa fornecida (lagartas da soja, do milho ou da cana, além de T. molitor e mosca doméstica) afetou significativamente estes parâmetros. Isso pode ser devido à alimentaçáo bem mais diversificada, envolvendo diferentes espécies.

Os períodos de pré e pós-oviposição de P. nigrispinus semelhantes nas diferentes dietas indicam que estes parâmetros são pouco afetados. Isso pode ser verificado em diversos trabalhos em laboratório (Holtz et al., 2010; Lemos et al., 2001) ou no campo (Oliveira et al., 2002b). Ambos os períodos de pré e pós-oviposição mais curtos permitem maior alocação de energia no período de oviposição (DE BorToli et al., 2011), que pode ser mais longo, como observado nas dietas com farelo de trigo e ração de galinha poedeira peletizada. Em consequência deste período mais longo verificado com essas dietas, também houve maior número de posturas. Isso permite um melhor desempenho reprodutivo do predador, confirmando as suas adequaçóes.

Não houve diferença significativa no período embrionário. Este, normalmente, é característico de cada espécie, sofrendo pouca influência de tratamentos nutricionais para sua criação, a não ser que haja diferenças bruscas na temperatura (SANTOS et al., 2004).

A viabilidade dos ovos foi semelhante entre os tratamentos. No entanto, este parâmetro normalmente é bastante sensível à alimentaçāo das formas imaturas (EvANGELISTA JÚNIOR et al., 2004; Freitas et al., 2006; Holtz et al., 2009; Oliveira et al., 2002a; VACARI et al., 2007), especialmente se houver ganho de peso de adultos (ZANUnCio et al., 2002b).

O número de posturas de P. nigrispinus no tratamento farelo de trigo foi maior que o encontrado em outros trabalhos que utilizaram T. molitor (larva ou pupa) como presa para esse predador. No entanto, os demais tratamentos apresentaram valores semelhantes aos observados nesses trabalhos (Oliveira et al., 2004; Freitas et al., 2006; Castro et al., 2012). O número de ovos por postura de P. nigrispinus foi semelhante entre os tratamentos, e o mesmo foi observado em trabalhos em que T. molitor foi presa desse predador (Oliveira et al., 2004; Freitas et al., 2006; Castro et al., 2012), ou com A. gemmatalis como presa (Матоs Neto et al., 2002). O número de ovos por fêmea de P. nigrispinus, em todos os tratamentos, foi superior aos valores observados em outros trabalhos que utilizaram essa mesma presa (Oliveira et al., 2004; Freitas et al., 2006; De Bortoli et al., 2011). 
O maior número de ovos por fêmea com o farelo de trigo pode ser devido ao desenvolvimento diferenciado dos ovários desse predador, em relação à dieta da presa consumida (LEMOs et al., 2005). Segundo esses autores, fêmeas de P. nigrispinus alimentadas com dieta artificial apresentaram menor período de oviposição, menor número de posturas, ovos por postura e número de ovos por fêmea. Do contrário, ao se alimentar de larvas de Alabama argillacea Hubner, 1818 (Lepidoptera: Noctuidae), $M$. domestica ou T. molitor foram obtidos bons resultados de reprodução. Neste caso, o tipo de presa, ou dieta artificial, afetou o desenvolvimento e a morfometria dos ovaríolos das fêmeas de P. nigrispinus. Fêmeas tratadas com as presas apresentaram ovaríolos com grande número de ovócitos em avançado ou intermediário estágio de desenvolvimento. Já as fêmeas alimentadas com a dieta artificial podem apresentar ovários atrofiados e ovaríolos sem ovócitos (Lemos et al., 2006). Portanto, o tipo de dieta do predador pode afetar seus órgãos de reprodução.

A semelhança da longevidade entre machos e fêmeas de P. nigrispinus alimentados com $T$. molitor indica que a fonte nutricional dessa presa não influência essa variável reprodutiva. Porém, a diversificação das espécies de presas como alimentos para P. nigrispinus pode influenciar em sua longevidade, como verificado em Oliveira et al., (2004).

Informações sobre a constituição de dietas de presas oferecidas para criaçóes de predadores têm sido estudadas nos últimos anos (Finke, 2002; Michaud, 2000; OonincX; Dierenfeld, 2012). Estes dados fornecem bases para a formulação e/ou adequação das dietas oferecidas, com o intuito de otimizar o desenvolvimento e adaptação dessas criações em laboratório.

\section{CONCLUSÃO}

A presa T. molitor, alimentada com todas as dietas, promoveu o crescimento da população de P. nigrispinus. Larvas de T. molitor alimentadas com fubá de milho não se mostraram adequadas para a criação de $P$. nigrispinus. A ingestão de larvas alimentadas com farelo de trigo proporcionou maior número total de ovos em fêmeas de $P$. nigrispinus, sendo esta dieta indicada para a criação da presa desse predador. Há necessidade de estudos mais detalhados sobre ecologia nutricional de presas alternativas. Isso permite aumentar a produção de criaçóes massais de percevejos predadores para uso em programas de controle biológico.

\section{AGRADECIMENTOS}

Ao Conselho Nacional de Desenvolvimento Científico e Tecnólogico (CNPq), à Coordenação de Aperfeiçoamento de Pessoal de Nível Superior (CAPES) e à Fundação de Amparo à Pesquisa do Estado de Minas Gerais (FAPEMIG) pelo apoio financeiro.

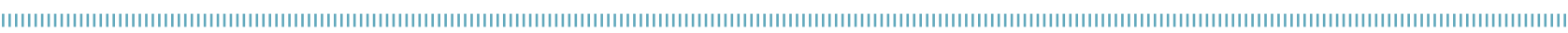

\section{REFERÊNCIAS}

ANDRADE, G.S.; SERRÃO, J.E.; ZANUNCIO, J.C.; ZANUNCIO, T.V.; LEITE, G.L.D.; POLANCZYK, R.A. Immunity of an alternative host can be overcome by higher densities of its parasitoids Palmistichus elaeisis and Trichospilus diatraeae. Plos One, v.5, p. 1-7, 2010.

CASTRO, A.A.; LACERDA, M.C.; ZANUNCIO, T.V.; RAMALHO, F.S.; POLANCZYK, R.A.; SERRÃO, J.E.; ZANUNCIO, J.C. Effect of the insect growth regulator diflubenzuron on the predator Podisus nigrispinus (Heteroptera: Pentatomidae). Ecotoxicology, v.21, p.96-103, 2012.

DE BORTOLI, S.A.; OTUKA, A.K.; VACARI, A.M.; MARTINS, M.I.E.G.; VOLPE, H.X.L. Comparative biology and production costs of Podisus nigrispinus (Hemiptera: Pentatomidae) when fed different types of prey. Biological Control, v.58, p.127-132, 2011.

ESPINDULA, M.C.; ZANUNCIO, J.C.; ANDRADE, G.S.; PASTORI, P.L.; OLIVEIRA, H.N.; MAGEVSKI, G.C. Desenvolvimento e reprodução de Podisus nigrispinus (Heteroptera: Pentatomidae) alimentado com lagartas de Heliothis virescens (Lepidoptera: Noctuidae). Idesia, v.28, p.17-24, 2010.

EVANGELISTA JÚNIOR, W.S.E.; GONDIM JÚNIOR, M.G.C.; TORRES, J.B.; MARQUES, E.J. Fitofagia de Podisus nigrispinus em algodoeiro e plantas daninhas. Pesquisa Agropecuária Brasilera, v.39, p.413420, 2004.

FERREIRA, J.A.M.; ZANUNCIO, J.C.; TORRES, J.B.; MOLINARUGAMA, A.J. Predatory behaviour of Podisus nigrispinus (Heteroptera: Pentatomidae) on different densities of Anticarsia gemmatalis (Lepidoptera: Noctuidae) larvae. Biocontrol Science and Technology, v.18, p.711-719, 2008.

FINKE, M.D. Complete nutrient composition of commercially raised invertebrates used as food for insectivores. Zoo Biology, v.21, p.269-285, 2002.

FISCHER, O.A.; MATLOVA, L.; DVORSKA, L.; SVASTOVA, P.; PERAL, D.L.; WESTON, R.T.; BARTOS, M.; PAVLIK, I. Beetles as possible vectors of infections caused by Mycobacterium avium species. Veterinary Microbiology, v.102, p.247-255, 2004.

FONT, E.; MOLINA-BORJA, M. Ultraviolet reflectance of color patches in Gallotia galloti lizards from Tenerife, Canary Islands. Institut Menorquí d'Estudis, v.8, p.201-221, 2004.

FRAENKEL, G.; BLEWETT, M.; COLES, M. The nutrition of the mealworm, Tenebrio molitor L. (Tenebrionidae, Coleoptera). Physiological Zoology, v.23, p.92-108. 1950. 
FREITAS, S.P.C.; EVANGELISTA JÚNIOR, W.S.; ZANUNCIO, J.C.; SERRÃO, J.E. Development, survival and reproduction of Podisus nigrispinus (Dallas, 1851) (Heteroptera: Pentatomidae) with salt and amino acids solutions supplementary diet. Brazilian Archives of Biology and Technology, v.49, p.449-455, 2006.

GRUNDY, P.R.; MAELZER, D.A.; BRUCE, A.; HASSAN, E. A massrearing method for the assassin bug Pristhesancus plagipennis (Hemiptera: Reduviidae). Biological Control, v. 18, p.243-250, 2000.

HOLTZ, A.M.; ALMEIDA, G.D.; FADINI, M.A.M.; ZANUNCIO-JUNIOR, J.S.; ZANUNCIO, T.V.; ZANUNCIO, J.C. Survival and reproduction of Podisus nigrispinus (Heteroptera: Pentatomidae): effects of prey scarcity and plant feeding. Chilean Journal of Agricultural Research, v.69, p.468-472, 2009.

HOLTZ, A.M.; PALLINI, A.; VENZON, M.; ZANUNCIO, J.C.; PRATISSOLI, D.; BARBOSA, W.F.; ANDRADE, G.S. Antibiosis of Eucalyptus plants on Podisus nigrispinus. Phytoparasitica, v.38, p.133-139. 2010.

INAYAT, T.P.; RANA, S.A.; RANA, N.; RUBY, T.; SIDDIQI, M.J.I.; KHAN, M.N.A. Predator-prey relationship among selected species in the croplands of central punjab, Pakistan. Pakistan Journal of Agricultural Sciences, v.48, p.153-157, 2011.

JAPYASSÚ, H.F; JOTTA, E.G. Forrageamento em Achaearanea cinnabarina levi 1963 (Araneae, Theridiidae) e evolução da caça em aranhas de teia irregular. Biota Neotropica, v.5, p.53-67, 2005.

JUSSELINO FILHO, P., ZANUNCIO, J.C., GUEDES, R.N.C.; FRAGOSO, D.B. Desarrollo y reproducción del depredador Brontocoris tabidus (Hemiptera: Pentatomidae) alimentado con larvas de Tenebrio molitor (Coleoptera: Tenebrionidae). Revista Colombiana de Entomologia, v.27, p.45-48, 2001.

LEMOS, W.P.; MEDEIROS, R.S.; RAMALHO, F.S.; ZANUNCIO, J.C. Effects of plant feeding on the development, survival and reproduction of Podisus nigrispinus (Dallas) (Heteroptera: Pentatomidae). International Journal of Pest Management, v.47, p.89- 93, 2001.

LEMOS, W.P.; RAMALHO, F.S.; SERRÃO, J.E.; ZANUNCIO, J.C. Effects of diet on development of Podisus nigrispinus (Dallas) (Het., Pentatomidae), a predator of the cotton leafworm. Journal of Applied Entomology, v.127, p.389-395, 2003.

LEMOS, W.P.; RAMALHO, F.S.; SERRÃO, J.E.; ZANUNCIO, J.C. Morphology of Female Reproductive Tract of the Predator Podisus nigrispinus (Dallas) (Heteroptera: Pentatomidae) Fed on Different Diets. Brazilian Archives of Biology and Technology, v.48, p.129-138, 2005.

LEMOS, W.P.; RAMALHO, F.S.; SERRAO, J.E.; ZANUNCIO, J.C.; BAUCE, E. Diet affects reproduction and number of oocytes per ovary of the predator Podisus nigrispinus (Dallas) (Heteroptera : Pentatomidae). Animal Biology, v.56, p.279-287, 2006.

LOPES, C.P.; VILLELA, G.G. Trealose e trealase em Tenebrio molitor L. Memórias do Instituto Oswaldo Cruz, v.70, p.577-582, 1972.

MAIA, A.H.N.; LUIZ, A.J.B.; CAMPANHOLA, C. Statistical inference on associated fertility life table parameters using Jackknife techinique:
Computational Aspects. Journal of Economic Entomology, v.93, p.511-518, 2000 .

MARTINSON, T.J.; FLASPOHLER, D.J. Winter bird feeding and localized predation on simulated bark-dwelling arth. Wildlife Society Bulletin, v.31, p.510-516, 2003.

MATOS NETO, F.C.; ZANUNCIO, J.C.; PICANÇO, M.C.; CRUZ, I. Reproductive characteristics of the predator Podisus nigrispinus fed with an insect resistant soybean variety. Pesquisa Agropecuária Brasileira, v.37, p.917-924, 2002.

MAYNTZ, D.; TOFT, S.; VOLLRATH, F. Effects of prey quality and availability on the life history of a trap-building predator. Oikos, v. 101, p.631-638, 2003.

MCCARTNEY, J.; STRINGER, I.A.N.; POTTER, M.A. Feeding activity in captive New Zealand lesser short-tailed bats (Mystacina tuberculata). New Zealand Journal of Zoology, v.34, p.227238, 2007.

MEDEIROS, R.S., RAMALHO, F.S., LEMOS, W.P., ZANUNCIO, J.C. Age-dependent fecundity and life-fertility tables for Podisus nigrispinus (Dallas) (Het., Pentatomidae). Journal of Applied Entomology, 124, 319-324. 2000.

MENEZES, C.W.G.; SANTOS, J.B.; ASSIS JÚNIOR, S.L.; SOARES, M.A.; FRANÇA, A.C.; FONSECA, A.J.; FERNANDES, A.F. Seletividade de atrazine e nicossulfuron a Podisus nigrispinus (Heteroptera: Pentatomidae). Planta Daninha, v.30, p.327-334, 2012a.

MENEZES, C.W.G.; SOARES, M.A.; SANTOS, J.B.; ASSIS JÚNIOR, S.L.; FONSECA, A.J.; ZANUNCIO, J.C. Reproductive and toxicological impacts of herbicides used in Eucalyptus culture in Brazil on the parasitoid Palmistichus elaeisis (Hymenoptera: Eulophidae). Weed Research, v.52, p.520-525, 2012 b.

MICHAUD, J.P. Development and reproduction of ladybeetles (Coleoptera: Coccinellidae) on the citrus aphid Aphis spiraecola Patch and Toxoptera citricida (Kirkaldy) (Homoptera: Aphididae). Biological Control, v. 18, n.3, p.287-297, 2000.

MORALES-RAMOS, J.A.; ROJAS, M.G.; SHAPIRO-ILAN, D.I.; TEDDERS, W.L. Self-selection of two diet components by Tenebrio molitor (Coleoptera: Tenebrionidae) larvae and its impact on fitness. Environmental Entomology, v.40, p.12851294, 2011.

MORALES-RAMOS, J.A.; ROJAS, M.G.; SHAPIRO-ILAN, D.I.; TEDDERS, W.L. Developmental plasticity in Tenebrio molitor (Coleoptera: Tenebrionidae): analysis of instar variation in number and development time under different diets. Journal of Entomological Science, v.45, p.75-90, 2010.

MOREIRA, L.A.; ZANUNCIO, J.C.; MOLINA-RUGAMA, A.J. Dados Biológicos de Podisus nigrispinus (Dallas) Alimentado com a Lagarta do Maracujazeiro Dione juno juno (Cramer). Anais da Sociedade Entomológica do Brasil, v.27, p.645-647, 1998.

MOURA, L.A; GRAZIA, J. Record of Podisus nigrispinus (Dallas) (Hemiptera: Pentatomidae) preying on Metrogaleruca obscura degeer (Coleoptera: Chrysomelidae). Neotropical entomology, v.40, p.619-621, 2011. 
NEVES, R.C.S.; TORRES, G.B.; ZANUNCIO, J.C. Production and storage of mealworm beetle as prey for predatory stinkbug. Biocontrol Science and Technology, v.20, p.1013-1025, 2010.

NG, W.K.; LIEW, F.L.; ANG, L.P.; WONG, K.W. Potential of mealworm (Tenebrio molitor) as an alternative protein source in practical diets for African catfish, Clarias gariepinus. Aquaculture Research, v.32, p.273-280, 2001.

OLIVEIRA, H.N.; ESPINDULA, M.C.; DUARTE, M.M.; PEREIRA, F.F.; ZANUNCIO, J.C. Development and reproduction of Podisus nigrispinus (Hemiptera: Pentatomidae) fed with Thyrinteina arnobia (Lepidoptera: Geometridae) reared on guava leaves. Brazilian Archives of Biology and Technology, v.54, p.429-434, 2011.

OLIVEIRA, H.N; PRATISSOLI, D.; PEDRUZZI, E.P.; ESPINDULA, M.C. Desenvolvimento do predador Podisus nigrispinus alimentado com Spodoptera frugiperda e Tenebrio molitor. Pesquisa Agropecuária Brasileira, v.39, p.947-951, 2004.

OLIVEIRA, J.E.M; TORRES, J.B.; CARRANO-MOREIRA, A.F.C.; BARROS, R. Efeito das Plantas do Algodoeiro e do Tomateiro, como Complemento Alimentar, no Desenvolvimento e na Reprodução do Predador Podisus nigrispinus (Dallas) (Heteroptera: Pentatomidae). Neotropical Entomology, v.31, p.101-108, 2002a.

OLIVEIRA, J.E.M.; TORRES, J.B.; CARRANO-MOREIRA, A.F.; RAMALHO, F.S. Biologia de Podisus nigrispinus predando lagartas de Alabama argillacea em campo. Pesquisa Agropecuária Brasileira, v.37, p.7-14, $2002 b$.

OONINCX, D.G.A.B.; DIERENFELD, E.S. An investigation into the chemical composition of alternative invertebrate prey. Zoo Biology, v.31, p.40-54, 2012.

SAEG. Sistema para Análises Estatísticas, Versão 9.1: Fundação Arthur Bernardes - UFV - Viçosa, 2007.

SANTOS, G.P.; ZANUNCIO, T.V.; RIBEIRO, G.T.R.; SILVA, E.P.; ZANUNCIO, J.C. Influência da temperatura no desenvolvimetno ninfal de Podisus distinctus (Dallas) Heteroptera Pentatomidae). Cerne, v.10, p.213-221, 2004.

SOUTHWOOD, T.R.E. Ecological methods with particular reference to the study of insect populations. 2a. ed. London: Chapman and Hall, 1978. 500p.

TORRES, J.B.; EVANGELISTA JR, W.S.; BARROS, R.; GUEDES, R.N.C. Dispersal of Podisus nigrispinus (Het., Pentatomidae) nymphs preying on tomato leafminer: Effect of predator release time, density and satiation level. Journal of Applied Entomology, v.126, p.326-332, 2002.
TORRES, J.B.; ZANUNCIO, J.C.; MOURA, M.A. The predatory stinkbug Podisus nigrispinus: Biology, ecology and augmentative releases for lepidoperan larval control in Eucalyptus in Brazil. CAB Reviews: Perspectives in Agriculture, Veterinary Science, Nutrition and Natural Resources, v.15, p.1-18, 2006.

URREJOLA, S.; NESPOLO, R.; LARDIES, M.A. Diet-induced developmental plasticity in life histories and energy metabolism in a beetle. Revista Chilena de Historia Natural, v.84, p.523-533, 2011.

VACARI, A.M.; OTUKA, A.K.; DE BORTOLI S.A. Desenvolvimento de Podisus nigrispinus (Dallas, 1851) (Hemiptera: Pentatomidae) alimentado com lagartas de Diatraea saccharalis (Fabricius, 1794) (Lepidoptera: Crambidae). Arquivos do Instituto Biológico, v.74, p.259-265, 2007.

VIVAN, L.M.; TORRES, J.B.; VEIGA, A.F.S.L.; ZANUNCIO, J.C. Comportamento de predação e conversão alimentar de Podisus nigrispinus sobre a traça-do-tomateiro. Pesquisa Agropecuária Brasileira, v.37, p.581-587, 2002.

ZAMPERLINI, B.; ZANUNCIO, J.C.; LEITE, J.E.M.; BRAGANÇA, M.A.L. Influência da alimentação de Tenebrio molitor L. 1758 (Coleoptera: Tenebrionidae) no desenvolvimento ninfal de Podisus connexivus Bergroth, 1891 (Hemiptera: Pentatomidae). Revista Árvore, v.16, p.224-203, 1992.

ZANUNCIO, J.C.; ALVES, J.B.; ZANUNCIO, T.V.; GARCIA, J.F. Hemipterous predators of eucalypt defoliator caterpillars. Forest Ecology and Management, v.65, p.65-73, 1994.

ZANUNCIO, J.C.; GUEDES R.N.C.; OLIVEIRA, H.N.; ZANUNCIO, T.V. Uma década de estudos com percevejos predadores: conquistas e desafios. In: Controle biológico no Brasil: parasitoide e predadores. São Paulo: Manole, p.495-505, 2002a.

ZANUNCIO, J.C.; LACERDA, M.C.; ZANUNCIO JÚNIOR, J.S.; SILVA, A.M.C.; ESPÍNDULA, M.C. Fertility table and rate of population growth of the predator Suppuntius cincticeps (Heteroptera: Pentatomidae) on one plant of Eucalyptus cloeziana in the field. Annals of Applied Entomology, v. 144, p.357-361, 2004.

ZANUNCIO, J.C., MOLINA-RUGAMA, A.J.; SANTOS, G.P.; Ramalho, F.S. Effect of body weight on fecundity and longevity of the stinkbug predator Podisus rostralis. Pesquisa Agropecuária Brasileira, v.37, p. 1225-1230, 2002b.

ZANUNCIO, J.C.; PEREIRA, F.F.; JACQUES, G.C.; TAVARES, M.T.; SERRÃO, J.E. Tenebrio molitor Linnaeus (Coleoptera: Tenebrionidae), a new alternative host to rear the pupae parasitoid Palmistichus elaeisis Delvare and Lasalle (Hymenoptera: Eulophidae). The Coleopterists Bulletin, v.62, p.64-66, 2008. 